Prog. Neuro-Psychopharmacol. vol.3, pp.483-489.

Pergamon Press Ltd. Printed in Great Britain.

\title{
A REEXAMINATION OF APOMORPHINE INDUCED STEREOTYPY IN THE RAT IN LIGHT OF SELF ADMINISTRATION EXPERIMENTS
}

\author{
RICHARD J. KATZ and E. DUFF BAILEY \\ Mental Health Research Institute, Department of Psychiatry \\ University of Michigan Medical Center \\ Ann Arbor, MI, USA
}

(Final form, May 1979)

\begin{abstract}
1. Apomorphine is known to both elicit stereotypy and to support behavioral self administration. The present report examined a possible contribution of stereotypy to self administration.

2. Non-contingent intraperitoneal injection of apomorphine in adult male Sprague-Dawley rats immediately prior to their placement in an experimental chamber significantly elevated barpressing above operant rates.

3. This indicates a novel dopamine induced behavior which may play a role in determining self administration behavior.

4. The data suggest three interpretations of previous self administration experiments.
\end{abstract}

Keywords: apomorphine, dopamine, reinforcement, self-administration, stereotypy

\section{Introduction}

The dopamine (DA) hypothesis of reinforcement suggests that contingent occupation of D A receptors is sufficient to maintain operant behavior. Evidence for this hypothesis has been presented and reviewed in a number of recent publications (e.g., Crow, 1976; Davis and Smith, 1977; Gill et al, 1979; Wise et al, 1976) and will therefore be discussed only briefly. Some general conclusions about the hypothesis may be drawn from previous studies, however. The hypothesis is based on two experimental paradigms. The first of these, self stimulation of the brain, has shown that electrical activation of DA containing cells in the substantia nigra supports robust self stimulation (Crow, 1976; Herberg et al, 1976; Stein, 1978). Related studies indicate that manipulations which increase DA receptor activation (i.e., administration of drugs with agonist properties such as apomorphine) increase self stimulation while manipulations which lower DA receptor activation (administration of blockers) conversely lower self stimulation (Crow, 1976; Herberg et al, 1976). Perhaps the more persuasive evidence for DA involvement in reward comes from a second set of experiments in which animals self administer DA receptor agonists. Apomorphine self administration has been reported in several species and across a variety of doses (Baxter et al, 1974; Davis and Smith, 1977; Gill et al, 1979; Wise et al, 1976). While these findings have now been independently verified by several researchers, they have also been qualified in several ways. Examination of data from the initial self administration report indicates extensive variability of behavior across sessions as well as instances of non-monotonicity in the dose response pattern (see Fig. 1; Baxter et al, 1974). In another report only a minority of rats acquired a rate of bar pressing exceeding operant levels (W ise et al, 1976). In this study, priming was necessary to institute responding, and aversion was demonstrated as a consequence of drug administration (IV ise et al, 1976). Other studies have shown spontaneous cessation of a self administration habit after continued access (Gill et al, 1979). In this last study priming again was necessary for maintaining responding and self administration was characterized by the absence of total intake regulation and the presence of pronounced stereotypy, vocalization, and self mutilation (Gill 
et al, 1979). These findings concerning stereotypy, $\mathrm{pr}$ iming, and the absence of intake regulation suggest apomorphine administration may be a more complex phenomenon than originally anticipated. Accordingly, we have reexamined whether non-contingent activation of DA receptors produces a stereotyped behavioral pattern which might contribute to previously observed patterns of self administration i.e. whether apamorphine might elicit bar-pressing independent of behavioral contingencies. The rationale for this study is that animals enter a conditioning situation with an operant rate of bar-pressing above zero. If sufficient apamorphine were to be adninistered by the operant response level to elicit additionel bar-pressing, this might establish a positive feedback loop for additional responding and contribute to final response rates and patterns. The present study was conducted in order to assess the following: could noncont ingent apamorphine pretreatment maintain bar-pressing behavior. We demonstrate herein that noncontingent apamorphine in fact facilitates of bar-pressing.

\section{Material and Methods}

Subjects. Eleven adult male Sprague-Dawley rats 300-500g each were obtained locally (Charles River Farms, Portage, MI) and maintained upon ad libitum food (Teklad $4.0 \%$ fat rodent diet S0836, Teklad, Madison, WI) and tap water, and autamatically programmed lighting cycles of $12 \mathrm{~h}$ light $/ 12 \mathrm{~h}$ darkness ( 1 ights on $=8: 00-20: 00 \mathrm{~h}$ ).

Drugs. Apomorphine $\mathrm{HCl}$ was injected intraperitoneally in doses of $0,5,10,15$ and $20 \mathrm{mg} / \mathrm{kg}$ in sterile $0.9 \%$ sodium chloride vehicle. A standard $5 \mathrm{mg} / \mathrm{ml}$ solut ion was used throughout. Five subjects received a standard dose of $5 \mathrm{mg} / \mathrm{kg}$ across all experimental sessions, while the remainder received both the $5 \mathrm{mg} / \mathrm{kg}$ dose and the remaining doses. For both groups of subjects $24 \mathrm{~h}-48 \mathrm{~h}$ intervals separated all sessions. Assigment to either condition was randam. Initial dose levels were chosen based upon two considerations. On the one hand, a dose of 5-10 $\mathrm{mg} / \mathrm{kg}$ is considered a standard behaviorally effective dose for a variety of procedures (Barnes and Elther ington, 1973). Also, examination of earlier reports of apomorphine self adninistration indicated that $4-8 \mathrm{mg} / \mathrm{kg}$ was characteristically ingested during a self administration session (Baxter et al, 1974; Fig 1, Table 2).

Apparatus. Subjects were randomly assigned to one of two types of experimental chamber. This was done to permit a direct assessment of the importance of apparatus differences in the drug effect. In fact, however, no apparatus specific effects were noted and data were therefore combined for analysis. The first of these was a standard $19 \times 25 \times 23.5 \mathrm{~cm}$ plexiglas operant chamber (Scientific Products A-100) equipped with a centrally located $3.8 \times 1.9 \times .8 \mathrm{~cm}$ stainless steel lever with a $10 \mathrm{~g}$ operating requirement (BSR/LVE \#121-03). The lever was mounted $3 \mathrm{~cm}$ fram the cage floor. The second chamber was of our own manufacture and was a wooden chamber of approximately the same dimensions $(20 \times 25 \times 30 \mathrm{~cm})$ which contained a single $8.9 \mathrm{~cm}$ width lever located $6.7 \mathrm{~cm}$ fram the floor with an operating requirement of $25.0 \mathrm{~g}$. Once a subject was assigned to a given apparatus, it was then run in it consistently. Recording was on standard solid state modules (Coulbourn).

Procedure. The experiment consisted of three phases. In the first, the subject was placed in the apparatus for determination of operant response rate and habituation. Two to three sessions were run without injection, and 2 with vehicle injection. The reason for running both controls was to a) al low comparison with previous self administration experiments that typically did not begin with injections (2) and b) to control wi thin-subjects for any response eliciting properties of injection. In the second, experimental stage of the experiment, all subjects were injected with apomorphine and bar-pressing behavior was recorded. Three to five sessions were run in this stage. In the third one two additional control sessions involving saline injections were run after all drug testing to examine final baseline response rates and assure reversibility of drug effects. All sessions were 90 minutes.

Statistical analysis. Results were evaluated statistically through repeated measures Analysis of Variance in which a session's effect served as the major dependent variable. 
a. Effect of constant doses of apomorphine: $5 \mathrm{mg} / \mathrm{kg}$ repeatedly. The rats showed low initial rates of response over the first four control sessions. Total responses per session ranged between one and 236. Injection of apomorphine produced considerable increases in rate, and these declined during the final control session. Figure 1 presents a summary of these animals' performances and figure 2 presents representative individual within-session response records for both a high and moderate responder (actual sessions totals respectively for these subjects were 1580 and 224). Treatments by subjects analysis of variance (Dixon and Massey, 1969) upon fig. 1 indicated significant differences across conditions ( $F 10,40$ $=18.86 \mathrm{p}<.0005)$.

b. Effect of increasing doses of apomorphine: $5-20 \mathrm{mg} / \mathrm{kg}$. Figure 3 shows the dose response relationship, as determined for the remaining subjects. Again the drug increased responding (F10,50= $9.13 \mathrm{p}<.001$ ) with $10 \mathrm{mg} / \mathrm{kg}$ the maximally effective dose. Typical response records for 2 subjects receiving 15 and $20 \mathrm{mg} / \mathrm{kg}$ are presented in Fig. 4 (session totals respectively were 541 and 395).

\section{$\underline{\text { Discussion }}$}

In the present experiment rats given apomorphine showed a non-contingent increase in bar-pressing; this may be presumed to be a stereotyped response. Apomorphine elicited behavior was dose dependent and showed changes over repeated administration. The present findings demonstrate a novel behaviorally complex form of stereotypy, and thereby extend previous reports.

Two points might be noted regarding the response rates and patterns of the present experiment. Careful examination of Figs. 2 and 4 clearly points to considerable within-sessions response variability. Moreover, attention to Figs. 1 and 3 indicates a decline in apormorphine elicited responding with repeated testing or high doses. Previous reports of self administration suggest both phenomena deserve specific comment.

Self administration studies have found that bar-pressing for contingent apomorphine is regularly spaced within and across sessions (Baxter et al, 1974; Wise et al, 1976). The present patterns clearly differ, and several procedural factors may be responsible for this difference. Total drug consumption was equated for the present and previous designs. In the present study, however, the entire dose of apomorphine was given prior to the session.

In previous studies apomorphine was given in considerably smaller portions across a session. Low doses of apomorphine are known to selectively activate presynaptic autoreceptors which are behaviorally inhibitory (e.g., Strombom, 1975). Thus, the low doses of apomorphine of previous studies might have caused initial behavioral inhibition of an ongoing baseline of apomorphine elicited stereotypy. The self administration experiment may represent an algebraic sum of autoreceptor induced inhibition and postsynaptically mediated behavioral excitation which jointly determine behavioral outcome. In the present design on the other hand a large initial dose may mask otherwise seen dose specific inhibitory effects.

The lack of uniformity in responding across repeated sessions or with high doses also deserves comment. As noted, animals may spontaneously cease responding in self administration designs. The present findings are consistent in demonstrating a reduced effect across sessions. The mechanism of this effect is not established. On the one hand the dropping off of responding may be seen as a tolerance phenomenon, in which continual DA stimulation gradually produces a smaller effect. However, tolerance to DA stimulation is not widely observed. If anything, stereotypy to amphetamines, increases with increased doses and tests (Randrup and Munkvad, 1970; Randrup et al, 1975). This suggests the need for an examination of the behavioral specificity of stereotypy over tests. One finding with amphetamines which may be germane is that while certain specific forms of stereotypy increase, other behaviors, among them translational motor activity decrease (Randrup and Munkvad, 1970). It is possible that the final and most invariant form of apomorphine induced stereotypy precludes the motor activity and integration required for behaviorally complex goal directed activity. In fact observations of animals at the highest dose suggest they are immobile but for repeated fine movements of various extremities.

The present results suggest three interpretations of apomorphine reinforcement. The first of these is the traditional DA reinforcement hypothesis (Crow, 1976; David and Smith, 1977; Stein, 1978). DA is self administered because of its rewarding properties. Such a view cannot be dismissed, although it should perhaps be reconsidered in light of the subjectively unpleasant effects of this drug (e.g., Stein, 1978) and its established aversive properties (W ise et 21,1976 ). Such an interpretation cannot account for the 


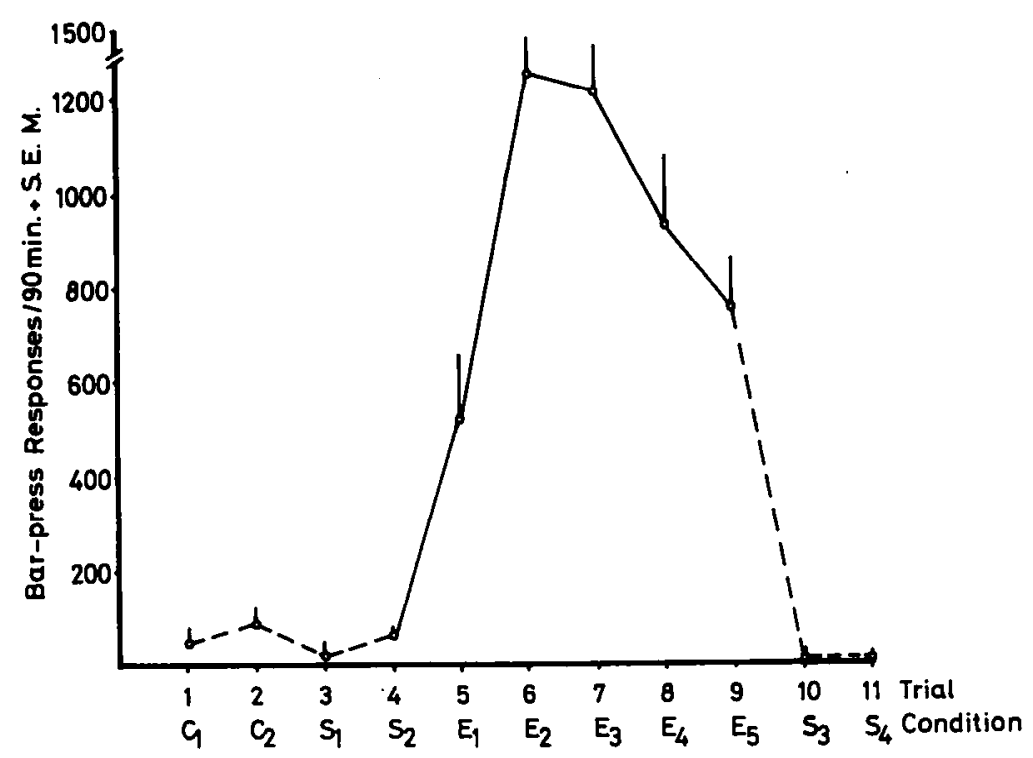

Fig. 1. Apomorphine ( $5 \mathrm{mg} / \mathrm{kg}$ ) elicitation of a complex behavioral stereotype (bar pressing) in the rat. C $=$ uninjected control session. $\mathbf{S}=$ saline injected control session. $\mathrm{E}=$ apomorphine injected session; session numbers given as subscripts. Injections immediately prior to the start of a session $(n=5)$.
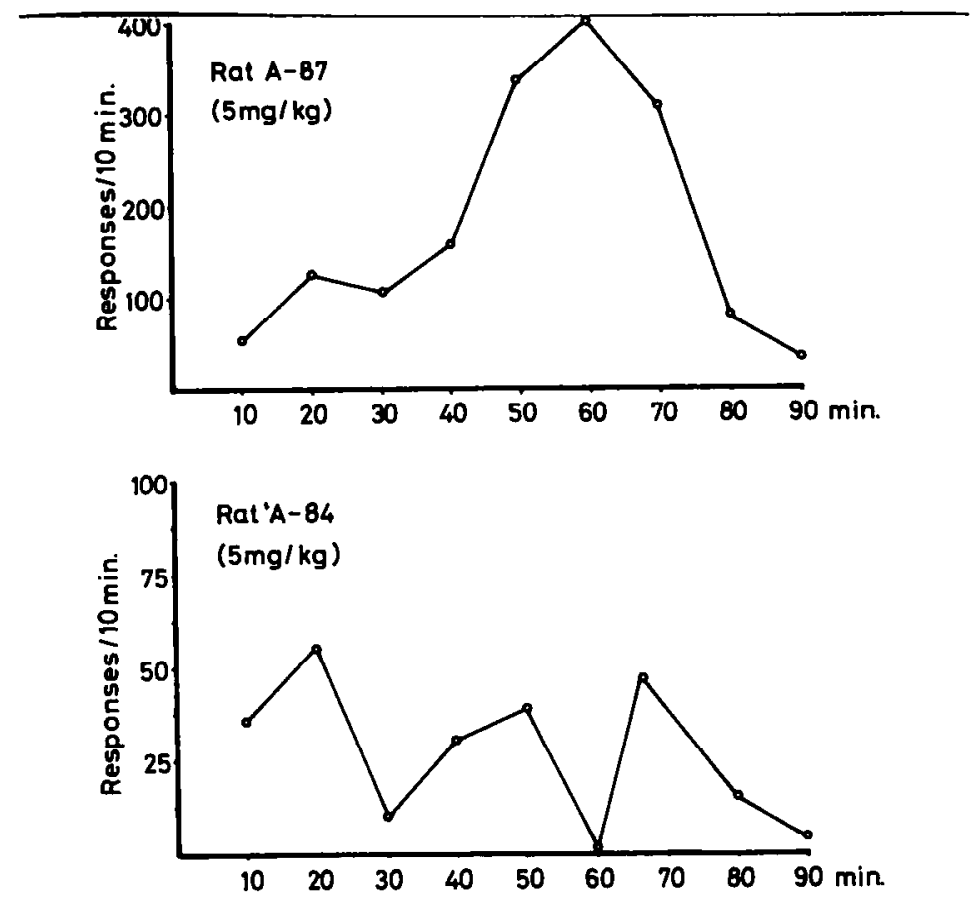

Fig. 2. Within session performance: apomorphine elicited bar-pressing. Individual records of two rats given apomorphine at the $5 \mathrm{mg} / \mathrm{kg}$ dose. 


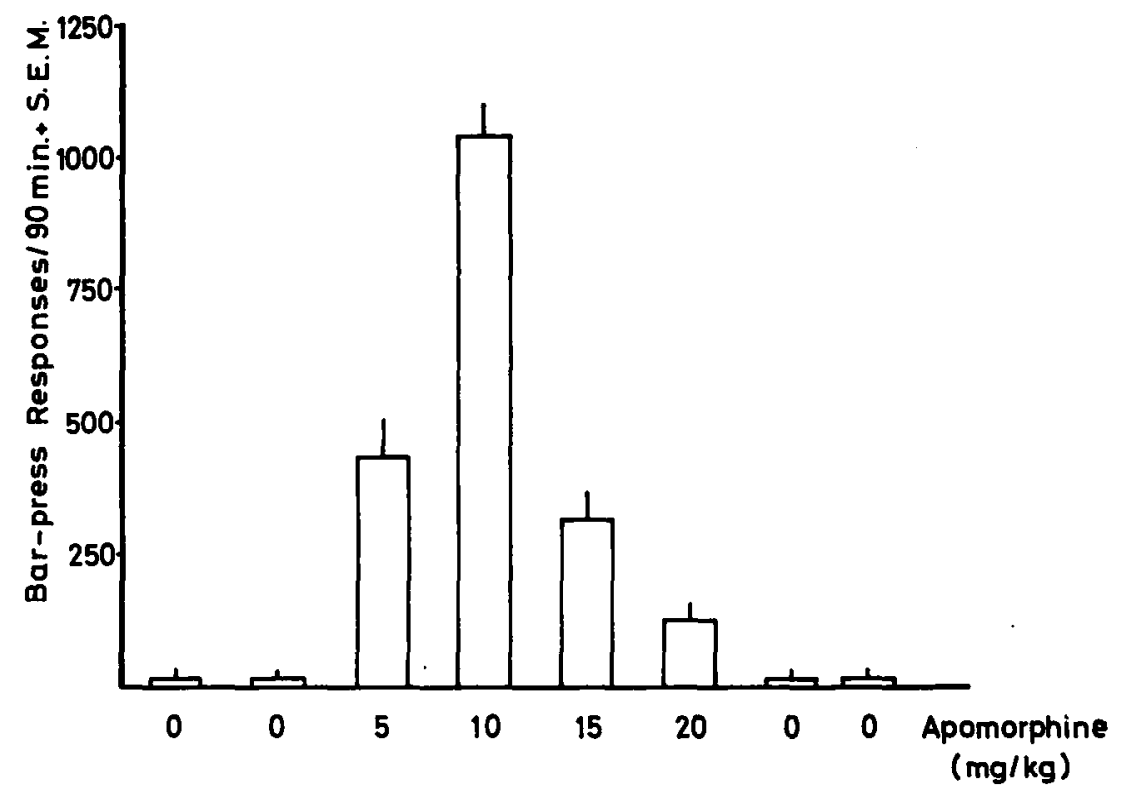

Fig. 3. Dose response curve for apomorphine elicited responding. Rats were injected immediately prior to placement in experimental chambers $(n=6)$.
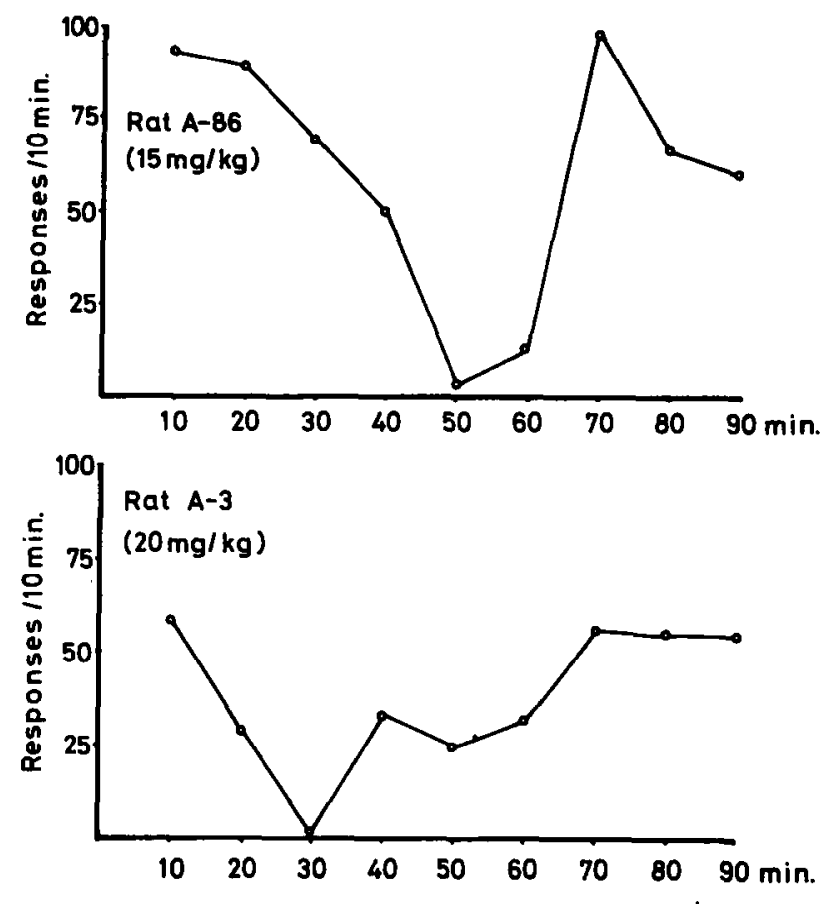

Fig. 4. Individual records for apomorphine elicited bar-pressing. Effect of high doses: 15 and $20 \mathrm{mg} / \mathrm{kg}$. 
present findings, and rather would suggest the observed response patterns are orthogonal to self administration responses and do not affect final response rates and patterns in self administration. The second interpretation suggests that one effect of apomorphine is the elicitation of a bar-pressing pattern of stereotypy. Operant rates of bar-pressing and positive feedback principles might then cause further drug taking. As suggested in the introduction if initial patterns of adventitious responding were to result in sufficient drug administration to support stereotypy, or if stereotypy were to cause further (stereotyped) barpressing then a pattern of self administration might occur without hedonic feedback. Such responding could in fact be further increased by possible classical conditioning of drug effects to distinctive apparatus cues, which could later elicit behavior by a learned association. While the idea of a behaviorally complex stereotype such as bar-pressing has not received wide consideration to date, results from experiments with amphetamines suggest it deserves additional examination. In the rat, for example, amphetamine induced bar-press stereotypy may interact with and facilitate operant behavior, and in primates stereotyped behaviors may include complex patterns of grooming and integrated motor rituals (Rendrup and Munkvad, 1970; Randrup et al, 1975). In humans amphetamines induce cognitive stereotypy "pundning" and such complex actions as repetitively cleaning a house or assembling and disassembling a motor (Randrup and Munkvad, 1970; Randrup et al, 1975). While amphetamines and apomorphine are different drugs, their ability to induce stereotypy may rest in the same system (Randrup and Munkvad, 1970; Randrup et al, 1975).

One final interpretation of the present and previous results is possible. Apomorphine may produce a motivated state which in turn causes psychomotor discharge, i.e., a drive to manipulate or behaviorally engage. This third explanation suggests an involvement of both previous explanations. On the one hanit, apomorphine is involved in reward, not directly, but indirectly through the induction of a drive-like state the satisfaction of which might produce further drug administration. Apomorphine might induce a generalized syndrome of motor activation which is selectively channelled into a set of bar-pressing behaviors. The syndrome is not entirely stereotyped, however, since it possesses a motivational component involving drive reduction. This suggestion has been made previously in a different context (Robinson et al, 1967) and in fact, apomorphine injected rats will learn to perform an operant response to obtain an object upon which they can then gnaw (Robinson et al, 1967).

Perhaps the most conservative interpretation of all results to date including self administration experiments would suggest that bar-pressing for apomorphine (B) is a function of three variables: reward $(R)$, stereotypy (S), and manipulative drive $(M), \quad B=f(R, S, M)$ and that the empirical contributions of each factor remain to be individually demonstrated. The onus now rests upon those holding strictly hedonic interpretations of DA self administration to run yoked controls or possibly double yoked controls (Kimmel and Terrant, 1968) to demonstrate a contribution of reward above and beyond that of motor activation and stereotypy. While the individual contributions of reward and manipulative drive are more difficult to evaluate, the use of learning techniques (Robinson et al, 1967) may offer some insight to this problem.

\section{Conclusion}

In conclusion, we have demonstrated high rates of bar pressing based upon apomorphine pretreatment. These results are of interest both because they show that stereotypy may assume a variety of different forms, and also because this particular form of stereotypy might affect self administration. We have discussed three interpretations of how stereotypy might be so involved. These results suggest a potential novel determinant of dopamine self administration.

\section{Acknowledgements}

The first author was supported in part by postdoctoral grant MH07417 from the National Institute of Mental Health through the Mental Health Research Institute. We are grateful to Mary Sies, Elizabeth Romkema and Esther W ashington for editorial assistance. 
BARNES, C. D. and ELTHERINGTON, L. B. (1973) Drug Dosage in Laboratory Animals, A Handbook. University of California Press, Berkeley.

BAXTER, B. L., GLUCKMAN, M. I., STEIN, L. and SCERNI, R. A. (1974) Self injection of apomorphine in the rat: positive reinforcement by a dopamine receptor stimulant. Pharmacol. Biochem. Behav. 2: 387-391.

CROW, T. J. (1976) Specific monoamine systems as reward pathways: evidence for the hypothesis that activation of the ventral mesencephalic dopamine neurons and noradrenergic neurons of the locus coeruleus complex will support self-stimulation responding. In: Brain Stimulation Reward, A. Wagner and E. T. Rolls (eds.), pp. 211-237. North Holland Publishers, The Netherlands.

DAVIS, W. M. and SMITH, S. G. (1977) Catecholaminergic mechanisms of reinforcement: direct assessment by drug self administration. Life Sci., 20: 483-492.

DIXON, W. J. and MASSEY, JR., F. J. (1969) Introduction to Statistical Analysis. 3rd edn, 638 pp. MeGraw-Hill, New York.

GILL, C. A., HOLZ, W. C., ZIRKLE, C. L. and HILL, H. (1979) Pharmacological modification of cocaine and apomorphine self-administration in the squirrel monkey. In: NeuroPsychopharmacology. Proc. of the 10th CINP Congress, P. Deniker, C. Radouco-Thomas and A. Villeneuve (eds), pp. 14771484. Pergamon Press, Oxford.

HERBERG, L. J., STEPHENS, D. N. and FRANKLIN, R. B. J. (1976) Catecholamines and self stimulation: evidence suggesting a reinforcing role for noradrenaline and a motivating role for dopamine. Pharmacol. Biochem. Behav. 4: 575-582.

KIMMEL, H. D. and TERRANT, F. R. (1968) Bias due to individual differences in yoked designs. Behav. Res. Methods Instrumentation, 1: 11-14.

RANDRUP, A. and MUNKVAD, $\overline{\mathrm{I}}$. (1970) Biochemical anatomical and psychological investigations of stereotyped behavior induced by amphetamines. In: Amphetamines and Related Compounds, E. Costa and S. Garattini (eds.), 695-713. Raven Press, New York.

RANDRUP, A., MUNKVAD, I., FOG, R. and AYHAN, I. H. (1975) Catecholamines in activation, stereotypy, and level of mood. In: Catecholamines and Behavior, vol. 1, A. J. Friedhoff (ed.) 89-107. Plenum Press, New York.

ROBINSON, P., DALEY, M. and WOLFF, P. C. (1967) Apomorphine induced reinforcement. Psychonomic Sci., 7: 117-118.

STEIN, L. (1978) Reward transmitters, catecholamines, and opioid peptides. In: A Review of Psychopharmacology, A Second Decade of Progress, M. A. Lipton, A. DiMascio and K. Killam (eds.) 569-583. Raven Press, New York.

STROMBOM, U. (1975) On the functional role of pre- and postsynaptic catecholamine receptors in brain. Acta Physiol. Scand. Supp. 431, 1-43.

WISE, R. A., YOKEL, R. A. and DEWITT, H. (1976) Both positive reinforcement and conditioned aversion from amphetamine and from apomorphine in rats. Science, 191: 1273-1275.

Inquires and reprint requests should be addressed to:

Dr. R. J. Katz

Mental Health Research Institute

Department of Psychiatry

University of Michigan Medical Center

Ann Arbor, MI 48109, USA 